
\title{
25 Research Soure \\ Effect of Acupoint Catgut Embedding on Intestinal Flora in Rats with Ovariectomized Osteoporosis
}

\section{Na Shi}

Nanjing University of Chinese Medicine

\section{Chuqiong Zhang}

Nanjing University of Chinese Medicine

\section{Long Han}

Nanjing University of Chinese Medicine

Gang Ouyang ( $D$ Oygang111@163.com )

Jiangsu Province Geriatric Hospital https://orcid.org/0000-0003-3938-696X

\section{Research}

Keywords: Acupoint catgut embedding, Postmenopausal osteoporosis(PMOP), Intestinal flora, Shortchain fatty acids(SCFAs), Bone mineral density(BMD)

Posted Date: August 12th, 2020

DOI: https://doi.org/10.21203/rs.3.rs-52205/v1

License: (1) (1) This work is licensed under a Creative Commons Attribution 4.0 International License. Read Full License 


\section{Abstract}

Background: To evaluate the effect of acupoint embedding on intestinal flora in rats with ovariectomized osteoporosis.

Methods: Thirty-two 3-month-old female SD rats were randomized into four groups: Group A without operation or treatment, Group B treated with the sham operation, Group C treated with the operation and without any treatment, and Group E treated with operation and acupoint embedding therapy. The osteoporosis animal model was generated by removing bilateral ovaries of rats, and unilateral "Shenshu" (BL23) and "Zusanli" (ST36) of rats were selected for embedding therapy every time. The therapy was performed once every 1 week for 12 consecutive weeks.

Results: The bone mineral density of femur and tibia and the estrogen levels in Group E are statistically higher than those in Group $C(p<0.05)$. The Beta diversity analysis shows the difference in the diversity and abundance of flora between Group A and Group $C$ is statistically significant $(p<0.01)$. In Group $E$, the abundance of probiotics such as firmicutes and bacteroidetes at the phylum level, lactobacillaceae, lachnospiraceae, muribaculaceae, and bacteroidaceae at the family level, lactobacillus gasseri, lactobacillus reuteri, lactobacillus intestinalis, and bacteroides acidifaciens at the species level is higher than those in Group C. The contents of short-chain fatty acid in Group E are significantly higher than those in Group C $(p<0.05)$.

Conclusion: Acupoint catgut embedding can improve bone density and increase the estrogen level in rats with osteoporosis, which maybe attributed to regulating composition and abundance of intestinal flora, and increasing the content of short-chain fatty acids.

\section{Background}

Postmenopausal osteoporosis is a common clinical disease occurred in $25 \%-50 \%$ postmenopausal women, and is the main type of primary osteoporosis ${ }^{[1]}$. Most patients have symptoms, such as general weakness, bone pain, and fracture proneness ${ }^{[2]}$, which seriously affects patients' quality of life. It belongs to the category of "Guwei" and "Gubi" in traditional Chinese medicine. The study of acupoint catgut embedding mechanism for this disease mainly focuses on the improvement of bone density, estrogen level, and biochemical indicators of bone metabolism ${ }^{[3-5]}$. Recent studies have showed that intestinal flora can impact human bone metabolism and is associated with the occurrence of osteoporosis ${ }^{[6-8]}$. Herein, We propose to preliminarily explore the mechanism of acupoint catgut embedding therapy on postmenopausal osteoporosis by observing the changes of intestinal flora in ovariectomized rats.

\section{Materials And Methods}

\section{Animals}


32 healthy female SD rats aged 3 months, with body mass of 180 200 g, were purchased from Zhejiang Provincial Laboratory Animal Center [SPF grade, License Number: SCXK (Zhejiang) : 2014-0001]. The rats were fed in the experimental Animal Center of Nanjing University of Chinese Medicine (barrier environment), the ambient temperature was $21^{\circ} \mathrm{C} \sim 25^{\circ} \mathrm{C}$, the humidity was $40 \% \sim 60 \%$, and the lighting time was 12 hours a day. During the feeding period, the rats were free to eat and drink. The animal feeding and experimental program has passed the ethical review of Nanjing University of Chinese Medicine (No.201812A023).

\section{Modeling and Grouping}

After 1 week of adaptive feeding, the rats were randomly divided into four groups with 8 rats in each group. After the intraperitoneal anesthesia of $3 \%$ sodium pentobarbital $(0.1 \mathrm{~mL} / 100 \mathrm{~g})$, the local hair was cleaned, a modified dorsal incision located at the intersection of a transverse finger on the lower margin of the lowest costal margin and a semi-transverse finger on the side of the lumbar spine was chosen. Pink ovaries could be found in the deep fat layer after a longitudinal incision of $0.8-1 \mathrm{~cm}$. Then the ovaries were removed after ligation at the uterine angle followed by suturing layer by layer. In Group B, the sham operation was conducted by only removing a small amount of adipose tissue around the ovary. To avoid the effect of antibiotics on experimental data, no antibiotics were used systematically or locally after the surgery. The rats were given food and water freely after the operation.

\section{Intervention Methods}

Rats in Group A, Group B, and Group C were fed normally without any intervention, while those in Group D were intervened with catgut embedding in "Shenshu"(BL23) and "Zu Sanli"(ST36). The location method of "Shenshu" and "Zusanli" were referred to "Experimental Acupuncture" . "Shen Shu" : lower sides of the 2nd lumbar vertebra; "Housanli" : posterolateral knee joint, about $5 \mathrm{~mm}$ under the fibula head.

Size 7 embedding needle (Gao Guan brand, production license: Su Food and Drug Administration Production License: 2001-0244) and 3/0 collagen line (Longteng Biological Company, production license: Gan Food and Drug Administration Production License: 20160099) were used for acupoint catgut embedding.

One assistant helped to hold the mouse and tighten local skin at acupoint. The performer hold needle by right hand and pierced the skin quickly with bevel up, the collagen wire were put into the body when the needle pierced to the required depth, then exited the needle. When the needle back to subcutaneous, pulled out of the needle and pressed the pinhole quickly, making sure that the line was placed in the muscle below the acupoint and no thread on the outside of the skin. Unilateral "Shenshu" and "Zusanli" of rats were selected each time and bilateral acupoints were alternated. The intervention was performed once every week for 12 consecutive weeks.

\section{Specimen Collection}


The rats were fasting for food but with free access to water after $8 \mathrm{pm}$ before specimens collection. On the second day, the rats were placed on the aseptic operating table after abdominal anesthesia. After the blood was withdrawn from the abdominal aorta, the serum was centrifuged and stored in the refrigerator at $-20^{\circ} \mathrm{C}$. The rat intestinal feces were collected with sterile tweezers and stored at $-80^{\circ} \mathrm{C}$ refrigerator. The right femur and tibia of the rats were stripped off after the rats were executed, then the bones were wrapped with saline gauze and placed in the EP tube storing at $-20^{\circ} \mathrm{C}$ after the muscles, tendons and other tissues were completely removed.

\section{Reagents and Instruments}

MEDIX90 dual-energy X-ray bone density detector (Austow, France), Reiber centrifuge (Beijing Reiber centrifuge co., LTD.), Thermo QE mass spectrometer, Thermo Vanquish UHPLC, Thermo Hyperil Gold column, Thermo ST16R cryogenic centrifuge, Thermo TRACE 1310-ISQ LT GC-MS, RT-6000 enzyme marker instrument (Shenzhen Redu company). Genjet gel recovery Kit (Thermo Scientific), Ion Plus Fragment Library Kit 48 RXNS Library building Kit (Thermo Fisher). Pentobarbital sodium was purchased from Merck (Shanghai, China), methanol, formic acid, and other reagents were purchased from Thermo Fisher (USA), ELISA kits were purchased from Shanghai zymes biological company.

\section{Index Detection}

\section{Determination of Bone Mineral Density}

The isolated femur and tibia of the rats were placed at room temperature for 30 minutes before testing. The bone mineral density was measured by the MEDIX90 dual-energy X-ray bone density detector.

\section{Blood Index Detection}

The serum samples were placed at room temperature to melt and then tested with ELISA kits for estrogen, alkaline phosphatase, and osteocalcin.

\section{Detection of Intestinal Flora and Short-chain Fatty Acids}

The rat feces were analyzed by Beijing Nuohezhiyuan company for the intestinal flora and short-chain fatty acid.

Intestinal flora: DNA of the samples was extracted by the CTAB method, and the diluted DNA was analyzed by PCR using primers in the $16 \mathrm{~S}$ V4 region (515F and 806R). The PCR products were purified with $2 \%$ agglutinin gel and then recovered with the GeneJET gel recovery kit. Then, Ion Plus Fragment Library Kit 48 RXNS construction Kit was used to build the Library. After Qubit quantification and detection, Ion S5TMXL was used for computer sequencing.

Short-chain fatty acids: It takes appropriate amounts of acetic acid, propionic acid, butyric acid, isobutyric acid, valeric acid, iso-valeric acid, and caproic acid standard substance to make 14 mixed standard concentration gradients with diethyl ether as the solvent. The sample was added $15 \%$ 
phosphoric acid and isocaproic acid, and then mixed with diethyl ether and centrifuged for $10 \mathrm{~min}$. The supernatant was taken and analyzed by GC-MS using Agilent HP-INNOWAX capillary column.

\section{Statistical Analysis}

The data were analyzed by SPSS 22.0 statistical software. The measurement results are expressed as mean \pm standard deviation. Oneway-ANOVA was used for inter-group comparison, and paired t-test is used for pairwise comparison, $\mathrm{p}<0.05$ is considered statistically significant.

\section{Results}

\section{BMD Values, Content of Estrogen, Osteocalcin, and Alkaline Phosphatase}

Figure1-a shows the BMD of femur and tibia in rats, the comparison between Group A and Group C, Group B and Group C, Group C and Group E are all significant, $p<0.05$.

Figure2-b.c.d show the results of estrogen, osteocalcin, and alkaline phosphatase. The differences in estrogen levels between Group A and Group C ( $<<0.05)$, Group B and Group $C(p<0.01)$, and Group C and Group E ( $<0.05)$ are all significant. The comparison of osteocalcin between Group B and Group $C$ is significant $(p<0.05)$. Compared with Group C, Group A and Group B had statistically significant differences in alkaline phosphatase respectively $(p<0.05)$.

\section{Results Of Intestinal Flora}

Figure2-a shows the number of OTUs in every sample at different taxonomic levels. OTUs of each sample at different taxonomic levels all have certain distinction. As seen from the Venn diagram in Fig. 2-b, there are certain differences in the flora species composition of the four groups, including 1,396 species in Group A, 1,408 species in Group B, 1,389 species in Group C, and 1,353 species in Group E. There is no significant difference in species among the four groups by chi-square test $(p>0.05)$. There are 1,314 species shared by Group A and Group B, 1,302 species shared by Group A and Group C, 1,316 species shared by Group B and Group C, and 1,335 species shared by Group C and Group E. The number of species shared by Group $C$ and Group $E$ is the highest while is not statistically significant $(p>0.05)$ compared with the number of species shared by the other two groups.

Figure3(a-f) show the top 10 species of maximum abundance in each group at different taxonomic levels. At the phylum level, the abundance of firmicutes and bacteroidetes in Group $E$ is higher than that in Group C; however, the abundance of tenericutes, actinobacteria, and verrucomicrobia in Group $\mathrm{C}$ is higher than that in Group E. At the class level, the abundance of clostridia, gammaproteobacteria, and mollicutes in Group C is higher than that in Group E; nevertheless, the abundance of bacteroidia and bacilli in Group E is higher than that in Group C. At the order level, the abundance of clostridiales, 
erysipelotrichales, and desulfovibrionales in Group $\mathrm{C}$ is higher than that in Group $\mathrm{E}$, the abundance of bacteroidiales, lactobacillales, enterobacteriales, and bacillales in Group E is higher than in Group C. At the family level, the abundance of peptostreptococcaceae and ruminococcaceae in Group $\mathrm{C}$ is higher than that in Group E, the abundance of lactobacillaceae, lachnospiraceae, muribaculaceae, and bacteroidaceae in Group $\mathrm{E}$ is higher than that in Group C. At the genus level, the abundance of romboutsia, unidentified clostridiales, unidentified lachnospiraceae, unidentified ruminococcaceae in Group $\mathrm{C}$ is higher than that in Group $\mathrm{E}$, the abundance of lactobacillus, turicibacter, bacteroides, and alistipes in Group $\mathrm{E}$ is higher than that in Group C. At the species level, the abundance of escherichia coli, clostridium papyrosolvens, clostridium disporicum, and the abundance of rumen_bacterium_Nk4A214 in Group $\mathrm{C}$ is higher than that in Group $\mathrm{E}$, the abundance of lactobacillus gasseri, lactobacillus reuteri, lactobacillus intestinalis, bacteroides acidifaciens, and clostridium_sp_MC_40 in Group E is higher than that in Group C.

Alpha diversity analysis can reflect the abundance and diversity of OTUs in the groups. The results show certain differences in the median, dispersion degree, outliers, and other aspects of samples within the four groups by ACE index analysis(Fig. 2-e), Shannon index analysis(Fig. 2-C), and Simpson index analysis(Fig. 2-d), but the differences in abundance and diversity of species within the four groups are not statistically significant further through the t-test, Wilcox rank-sum test, and Tukey test $(p>0.05)$.

Beta diversity can evaluate the differences in the composition of flora from samples. By Weighted Unifrac-Wilcox test in Fig. 2-f, the differences between Group A and Group C $(p<0.01)$, Group A and Group $E(p<0.01)$, Group A and Group B $(p<0.01)$ are statistically significant, which indicates that the differences of Beta diversity within the four groups are statistically significant.

Under the Index heat map of Beta diversity(Fig. 3-g), the different coefficient between Group A and Group E is the smallest, while the different coefficients between Group A and Group C, Group B and Group C are

larger, which indicates that the diversity difference between Group A and Group $\mathrm{E}$ is the smallest.

In the UPGMA cluster tree, the two groups of samples with the highest similarity gather in priority to form a new node (a new sample), and then gather the new samples to other samples with the highest similarity. Repeatedly, all the samples are gathered together to eventually get a complete cluster tree. Figure 3-f shows that the similarity between Group A and Group $E$ is the highest at the phylum level, while Group $\mathrm{C}$ has the lowest similarity with other groups.

\section{Content Of Short-chain Fatty Acid}

Figure1-e.f show the results of intergroup comparison of the short-chain fatty acids. The differences between Group A and Group $C$ in all types of short-chain fatty acids are significant $(p<0.05$ or $p<0.01)$. The differences between Group $C$ and Group $E$ in acetic acid, butyric acid, isovaleric acid, valeric acid, and caproic acid are significant $(p<0.05)$. The comparison of butyric acid, isovaleric acid, and valeric acid between Group B and Group C are all significant $(p<0.05)$. 


\section{Discussion}

Decreased ovarian function and low estrogen levels after menopause are the main causes of postmenopausal osteoporosis ${ }^{[9]}$. Decreased estrogen levels can stimulate the release of inflammatory factors, thereby increasing the activity of osteoclasts and leading to osteoporosis ${ }^{[10]}$. Besides, decreased estrogen can also reduce the sensitivity of bone to mechanical stimulation, resulting in pathological changes similar to disuse bone loss ${ }^{[11]}$. Studies confirm that decreased estrogen can also reduce the diversity of intestinal flora ${ }^{[12-13]}$, and Intestinal flora can affect bone metabolism by releasing small molecules, such as estrogen analogs and serotonin, to participate in immune regulation and affect the absorption and metabolism of calcium and phosphorus ${ }^{[14-15]}$. Therefore, it can be considered that the development of PMOP is associated with the diversity of intestinal flora.

Acupoint catgut embedding is based on the theory of acupuncture and moxibustion. In the treatment, the collagen thread is embedded into the acupoint to generate continuous stimulation to the local area, so as to achieve the "long-acting needle sensation effect". Compared with conventional acupuncture, the effect lasts longer with a stronger stimulus. It can also avoid the pain of daily acupuncture and save time.

Studies have shown that acupoint catgut embedding can significantly improve the clinical symptoms of PMOP patients, regulate hormone levels, and increase bone density ${ }^{[16-17]}$. In our study, we also have found that the BMD and estrogen levels of rats in Group E were significantly increased, and the concentration of osteocalcin and alkaline phosphatase were increased too.

After the rats were ovariectomized, aseptic mice showed significant decrease in bone mass compared with control group, this meaned that the sterility of the mice could prevent the loss of bone trabeculae which was caused by steroid levels decreasing ${ }^{[18]}$. In our experiment, we also found that the diversity of intestinal flora decreased at different levels. After acupoint catgut embedding therapy, the diversity could be increased to some degree.

Intestinal flora includes probiotics, pathogenic bacteria, and opportunistic bacteria. Studies have found that probiotics such as aactobacillus, bifidobacterium, and escherichia colican, etc. can stimulate the intestinal cells to secrete glucose-dependent trypsin and glucagon-like peptide区, which can promote bone formation, inhibit bone absorption, and improve bone health ${ }^{[19-20]}$. In addition, taking a certain amount of probiotics can also improve bone strength and reduce the incidence of osteoporosis ${ }^{[21-23]}$. It has been reported $^{[24]}$ that firmicutes, including fusobacteria, play a role in regulating the immune system and can maintain host immune system homeostasis by up-regulating the proliferation and function of $\mathrm{T}$ cells. Our study showed that in Group $E$ the abundance of firmicutes and bacteroidetes at the phylum level, bacteroidia and bacilli at the class level, bacteroidiales, lactobacillales, enterobacteriales, and bacillales at the order level, lactobacillaceae, lachnospiraceae, muribaculaceae, and bacteroidaceae at the family level, lactobacillus, turicibacter, bacteroides, and alistipes at the genus level, lactobacillus gasseri, lactobacillus reuteri, lactobacillus intestinalis, and bacteroides acidifaciens at the species level is higher 
than those in Group C. These demonstrated that acupoint catgut embedding can regulate intestinal flora and improve the abundance of probiotics.

Pathogenic bacteria, such as proteobacteria and bacilli, have the opposite effect. On one hand, they can produce endotoxin to bind to toll-like receptors on the surface of host immune cells, causing inflammatory response to promote bone resorption ${ }^{[25]}$; on the other hand, they also produce serotonin which can enter the blood circulation to inhibit bone formation and reduce the percentage of bone trabecular volume ${ }^{[26]}$. Studies have shown ${ }^{[27]}$ that the number of gemmatimonadetes and chloroflexi in postmenopausal osteoporosis patients is higher than those in normal people. This is consistent with our results that in Group $C$ the abundance of tenericutes, actinobacteria, and verrucomicrobia at the phylum level; the abundance of clostridia, gammaproteobacteria, and mollicutes at the class level; the abundance of clostridiales, erysipelotrichales, and desulfovibrionales at the order level; the abundance of peptostreptococcaceae and ruminococcaceae at the family level; the abundance of romboutsia, unidentified clostridiales, unidentified lachnospiraceae, and unidentified ruminococcaceae at the genus level is all higher than those in Group E. These demonstrated that the abundance of pathogenic bacteria in ovariectomized rats can be decreased after acupoint catgut embedding intervention.

Moreover, anaerobic bacteria, such as bifidobacterium and bacteroidetes, can produce short-chain fatty acids(SCFAs) by fermenting undigested carbohydrates ${ }^{[28]}$. Studies have confirmed that colonization of intestinal flora in sterile mice can increase the concentration of SCFAs in the cecum ${ }^{[29-30]}$.

SCFAs mainly includes acetic acid, propionic acid, and butyric acid, etc ${ }^{[28]}$. Butyric acid can regulate acetylation of histone deacetylase, control epigenetic expression, and then induce the proliferation and differentiation of regulatory $T$ cells in the intestine to enhance immune response, and regulate osteoclast differentiation ${ }^{[31-32]}$. Butyrate can also provide energy for intestinal mucosal epithelial cells, improve intestinal villi structure, and increase absorption area so that facilitate intestinal calcium absorption ${ }^{[33]}$.

The increased content of short-chain fatty acids can reduce the $\mathrm{pH}$ value of the intestinal tract and decrease the complex formed by calcium ion and phosphine in the intestinal tract, thus increase calcium absorption ${ }^{[34]}$. Besides, short-chain fatty acids may promote calcium absorption through epigenetic regulation and signaling pathways affecting mineral absorption ${ }^{[34-35]}$. Short-chain fatty acids can also regulates the number and function of colonic Treg cells, indirectly affecting osteoclasts through $T$ cells formation ${ }^{[36]}$. On the whole, SCFAs has been shown to promote bone formation, but the expression of SCFAs receptor in different bone cell types has not been reported, whether intestinal flora needs to mediate bone remodeling through SCFAs receptor remains unclear ${ }^{[29]}$.

Our experiment showed that the content of SCFAs increased significantly after acupoint catgut embedding therapy compared with the untreated group, which may be achieved by increasing the abundance of probiotics.

\section{Conclusion}


In conclusion, PMOP is related to bone resorption process mediated by intestinal flora and estrogen deficiency, which is essentially an immune response stimulated by antigen. Acupoint catgut embedding is an effective treatment for PMOP. Its effect on increasing bone mineral density, improving estrogen level and other aspects may be attributed to the increase of probiotic abundance, the decrease of pathogenic bacteria abundance, and the adjustment of the level of SCFAs. However, the specific role of acupoint catgut embedding treatment played by regulating inflammatory factors and signaling pathways needs to be further studied in the future.

\section{Abbreviations}

PMOP

Postmenopausal osteoporosis; SCFAs:Short-chain fatty acids; BMD:Bone mineral density.

\section{Declarations}

\section{Acknowledgements}

Not applicable.

\section{Authors' contributions}

GOY conceived and designed the study. NS, CQZ and LH performed the experiments. NS analyzed the data and wrote the manuscript. All authors read and approved the final manuscript.

\section{Funding}

The present study was supported by Chinese medicine science and technology development project of Shandong province(2019-0855), Jiangsu provincial health department cadre health bureau subject(BJ16023), Six talent peak projects in Jiangsu province(2014-ww-013).

\section{Availability of data and materials}

The datasets analyzed during the present study are available from the corresponding author on reasonable request.

\section{Ethics approval and consent to participate}

The protocols of the present study were approved by the Ethics Committee of Nanjing University of Chinese medicine(Nanjing, China, no. 201812A023).

\section{Consent for publication}

We declare that the Publisher has the Author's permission to publish the relevant contribution. 


\section{Competing interests}

The authors declare that there is no conflict of interest regarding the publication of this paper.

\section{Author details}

${ }^{1}$ Department of Rehabilitation, Linyi People's Hospital, Linyi 276003, China; ${ }^{2}$ Department of Acupuncture and Moxibustion, Huai 'an TCM Hospital Affiliated to Nanjing University of Chinese Medicine, Huai 'an 223001, China; ${ }^{3}$ First Clinical College, Nanjing University of Chinese Medicine, Nanjing 210023, China; ${ }^{4}$ Department of Traditional Medicine, Jiangsu Province Geriatric Hospital, Nanjing 210024, China.

\section{References}

1. Hong XH, Lei W, Hong H: Introduction to experimental research on osteoporosis.

Guangzhou, China: Guangdong Science and Technology Publishing House, 2018.

2. Deng LW, Mu L, Liu Y: Distribution of TCM syndromes in 130 cases of postmenopausal osteoporosis. Journal of Chengdu University of Chinese Medicine. 2016; 39 (2) : 76-78.

3. Zhong PC, Peng NJ, Zhao XH. Therapeutic Effect of acupoint catgut embedding combined with Western medicine in the treatment of osteoporosis. Modern Journal of Integrated Traditional Chinese and Western Medicine.2018; 27 (16): 1714-1716.

4. Jia Sj, Chen J, Lei XH, et al. Effect of acupoint catgut embedding on bone mineral density in people at high risk of osteoporosis. ACTA Chinese Medicine.2018; 33 :(1)166-170.

5. Wang WQ. Effect of acupoint catgut embedding on bone density and related cytokines in ovariectomized rats. Journal of practical traditional Chinese medicine. 2017; 33(2):112-113.

6. Maynard CL, Elson CO, Hatton RD, et al: Reciprocal interactions of the intestinal microbiota and immune system. Nature. 2012; 489(7415): 231-241.

7. Goulet O: Potential role of the intestinal microbiota in programming health and disease. Nutr Rev. 2015; 73(Suppl 1): 32-40.

8. Brzozowska MM, Sainsbury A, Eisman JA, et al: Bariatric surgery, bone loss, obesity and possible mechanisms. ObesRev. 2013; 14(1): 52-67.

9. Zhong X, Zhao LH, Fan YS: Current status of research on prevention and treatment of postmenopausal osteoporosis by acupuncture. Asia Pacific traditional medicine. 2008; 4 (4) : 63-65.

10. Shen H, Wei X, Xie YM, et al: Study on the correlation between fracture risk factors and TCM symptoms in postmenopausal osteoporosis. Chinese journal of integrated traditional Chinese and 
western medicine. 2017; 37 (1) : 50-56.

11. Feng J, Liu S, Ma S, et al: Protective effects of resveratrol on postmenopausal osteoporosis: regulation of SIRT1-NF-kappa B signaling pathway. Acta Biochim Biophys Sin(Shanghai). 2014; 46: 1024-1033.

12. Breban M: Gut microbiota and inflammatory joint diseases $\square$ Joint Bone Spine Revue Du Rhumatisme. 2016; 83(6): 645-649ه

13. Flores R, Shi J, Fuhrman B,et al: Fecal microbial determinants of fecal and systemic estrogens and

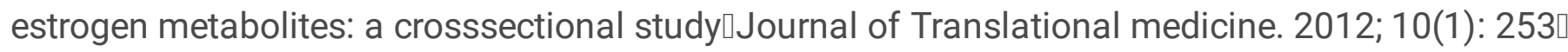

14. Mccabe L, Britton RA, Parameswaran N: Prebiotic and probiotic regulation of bone health: role of the intestine and its microbiome Curr Osteoporos Rep. 2015; 13(6): $363-710$

15. Lambert M N T, Thybo C B, Lykkeboe S, et al : Combined bioavailable isoflavones and probiotics improve bone status and estrogen metabolism in postmenopausal osteopenic women: A randomized controlled trial. Am J Clin Nutr. 2017; 106(3): 909-920.

16. Zhou XL, Hu MQ, Zhang Y, et al. Clinical efficacy of acupoint catgut embedding in the treatment of postmenopausal osteoporosis. Sichuan traditional Chinese medicine.2014; 32(7): 159-160.

17. Song YN, Li LH, Zhang LL, et al. Effect of acupoint catgut embedding on bone density and bone biomechanics in senile osteoporosis mice. Chinese journal of osteoporosis. 2015; 21(1):19-24.

18. Li JY, Chassaing B, Tyagi AM, et al. Sex steroid deficiency associated bone loss is microbiota dependent and prevented by probiotics. J Clin Invest. 2016; 126(6):2049-2063.

19. Mccabe L, Britton RA, Parameswaran N: Prebiotic and Probiotic Regulation of Bone Health: Role of the Intestine and its Microbiome. Curr Osteoporos Rep. 2015; 13: 363-3710

20. Campbell JE, Drucker DJ: Pharmacology, physiology, and mechanisms of incretin hormone action $\square$ Cell Metab. 2013; 17: 819-837ロ

21. Ohlsson C, Engdabl C, FAk F, et a1: Probiotics protect mice from ovariectomy-induced cortical bone loss $\square$ PLoS One. 2014; 9(3): e92368ם

22. Britton RA, Irwin R, Quach D, et a1: Probiotic L.reuteri treatment prevents bone loss in a menopausal ovariectomized mouse model[Journal of Cellular Physiology. 2014; 229(11): 1822-1830[

23. Parvaneh K, Ebrahimi M, Sabran MR, et al: Probiotics(Bifidobacterium longum)increase bone mass density and upregulate Spare and Bmp-2 genes in rats with bone loss resulting from ovariectomyロBiomed Research International. 2015; 2015: $10 \square$ 
24. Sjögren K, Engdahl C, Henning P, et al: The gut microbiota regulates bone mass in mice. Journal of Bone \& Mineral Research. 2012; 50(6): S91-S92

25. Villa CR, Ward WE, Comelli EM: Gut microbiota-bone axis $₫$ Crit Rev Food Sci Nutr. 2017; 57: 16641672》

26. Yadav VK, Ryu JH, Suda N, et al: Lrp5 controls bone formation by inhibiting serotonin synthesis in the duodenum[Cell. 2008; 135: 825-837ロ

27. Peng Y, Li XB: Spleen deficiency syndrome and intestinal microecology. World Chinese Journal of digestion. 2012;20 (34) : 3287-3291.

28. Morrison DJ, Preston T. Formation of short chain fatty acids by the gut microbiota and their impact on human metabolism. Gut Microbes. 2016;7(3):189-200.

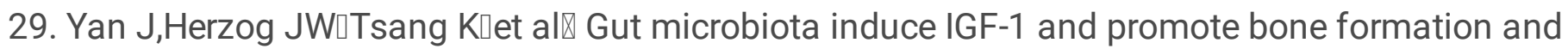
growth $\square$ Proc Natl Acad Sci USA囚2016; 113(47):E7554-E7563!

30. Smith PM, Howitt MR Panikov N囚 et al $\mathbb{T}$ The microbial metabolites $\square$ short-chain fatty acids $\square$ regulate colonic Treg cell homeostasis. Science. 2013; 341(6145):569-573ロ

31. Davie JR. Inhibition of histone deacetylase activity by butyrate. J Nutr. 2003; 133(7 Suppl)S2485S2493.

32. Arpaia N, Campbell C, Fan X, et al. Metabolites produced by commensal bacteria promote peripheral regulatory T-cell generation. Nature. 2013; 504(7480):451-455.

33. Donohoe DR, Garge N, Zhang X, et al: The microbiome and butyrate regulate energy metabolism and autophagy in the mammalian colon־Cell Metab. 2011; 13: 517-526!

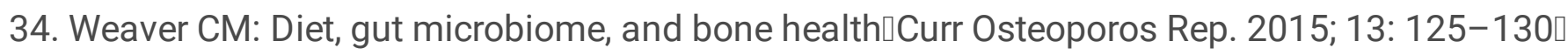

35. Yonezawa T, Kobayashi Y, Obara Y: Short-chain fatty acids induce acute phosphorylation of the p38 mitogen-activated protein kinase/heat shock protein 27 pathway via GPR43 in the MCF-7 human breast cancer cell line. Cell Signal. 2007;19: 185-193ロ

36. Smith PM, Howitt MR, Panikov N, et al: The microbial metabolites, short-chain fatty acids, regulate colonic Treg cell homeostasis. Science. 2013; 341(6145):569-573.

\section{Figures}



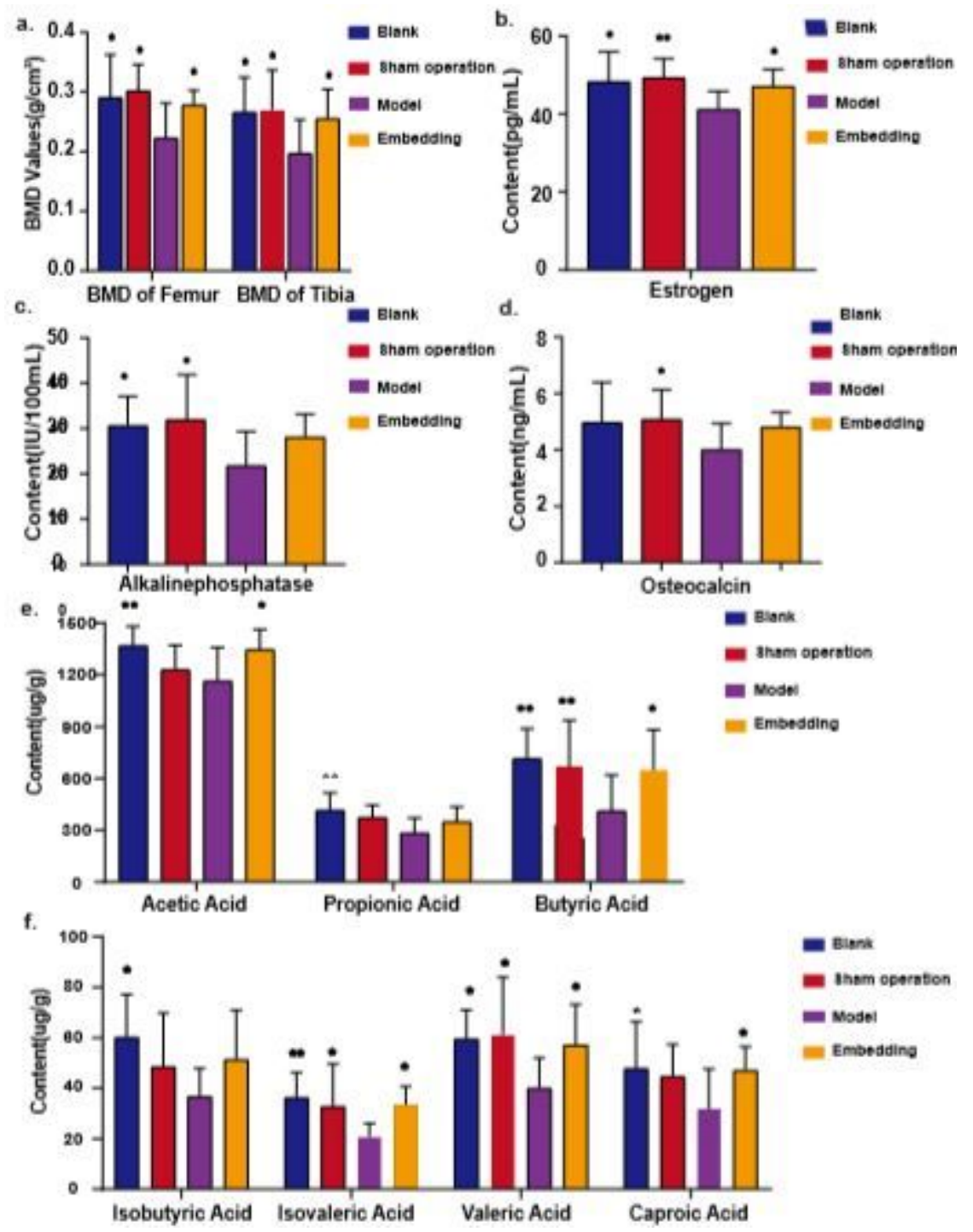

Figure 1

BMD, blood index and SCFAs of each group, data are shown as mean \pm SD $(n=8)$. *represents that $p$ $<0.05$ vs. model group, ${ }^{\star \star} p<0.01$. a.BMD values of femur and tibia. b.Content of estrogen. $b$. Content of alkalinephosphatase. c.Content of osteocalcin. d.Content of acetic acid,propionic acid,butyric acid. f.Content of isobutyric acid,isovaleric acid,valeric acid, caproic acid. 
a.

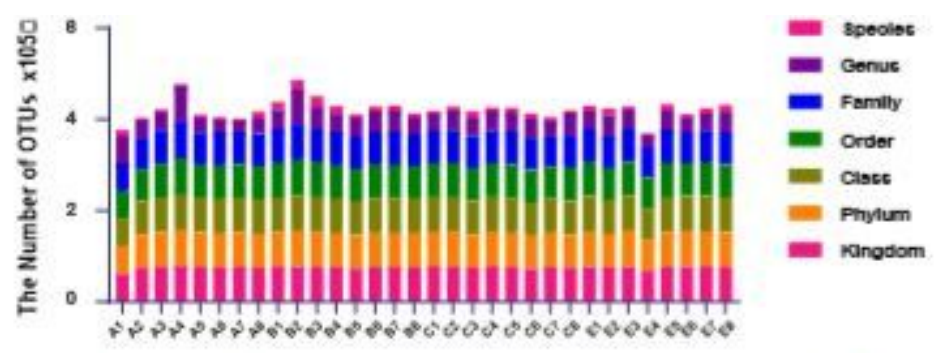

b.

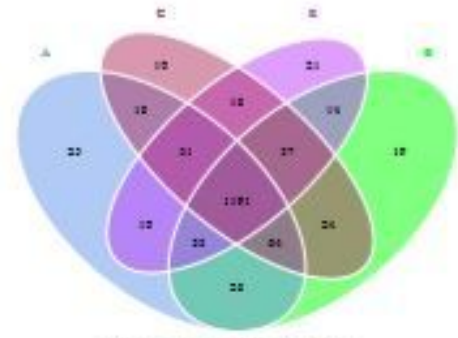

Venn diagram of OTUs

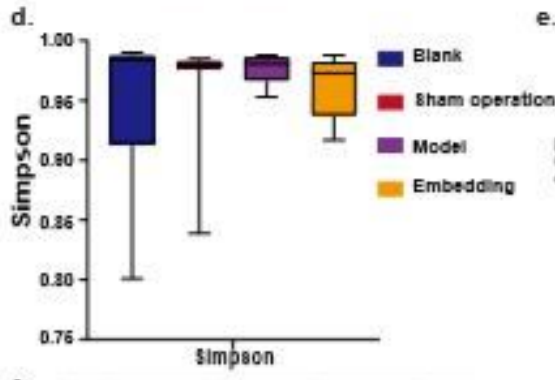

f.

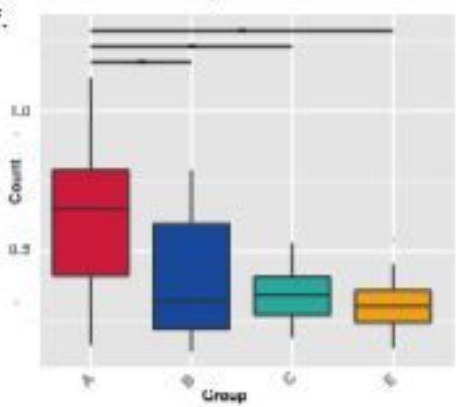

c.
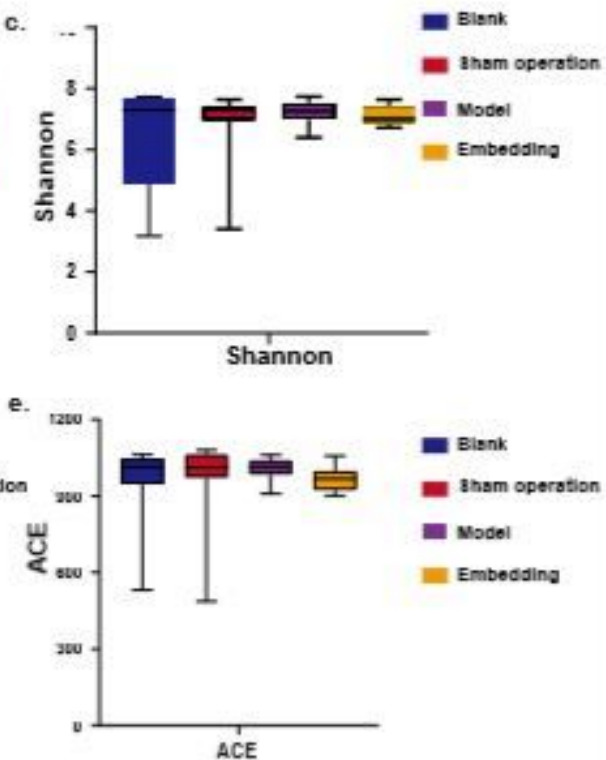

Fig 2. Relative abundance of species, venn diagram of OTUs and multiple comparative analysis. a. The numbe of OTUs at different levels of classification. b. Venn diagram of OTUs among four groups. c.d.e. Alpha diversity analysis among different groups(Shannon, Simpson, and ACE), the differences in abundance and diversity of species within the four groups are not statistically, $p>0.05$. $f$. Beta diversity analysis among different groups. -Compare between two groups $p<0.05, \cdots p<0.01$. Data are shown as mean \pm SD.

\section{Figure 2}

Relative abundance of species, venn diagram of OTUs and multiple comparative analysis. a. The numbe of OTUs at different levels of classification. b. Venn diagram of OTUs among four groups. c.d.e. Alpha diversity analysis among different groups(Shannon, Simpson, and ACE), the differences in abundance and diversity of species within the four groups are not statistically, $p>0.05$. f. Beta diversity analysis among different groups. ${ }^{*}$ Compare between two groups $p<0.05, * \star p<0.01$. Data are shown as mean \pm SD. 

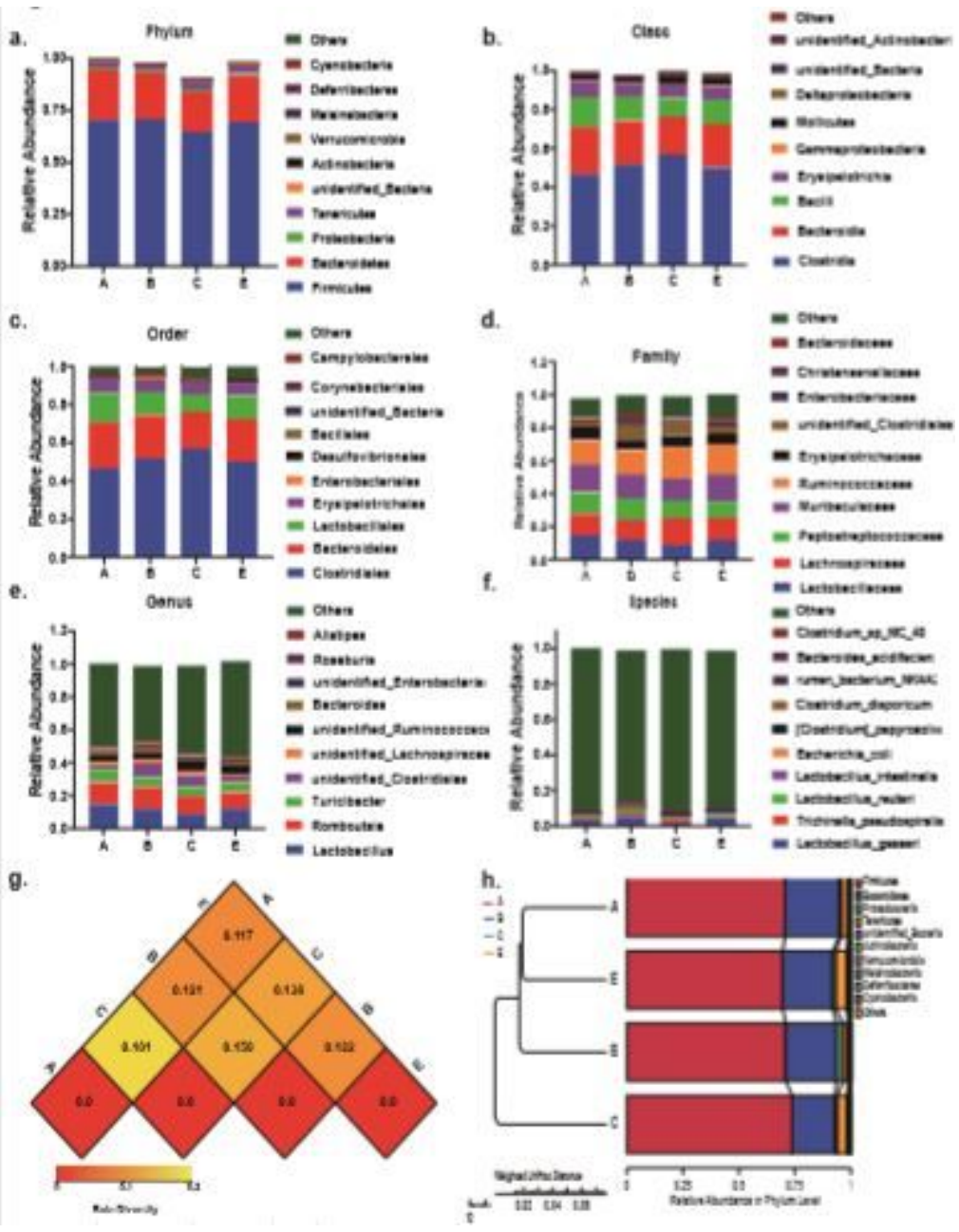

\section{Figure 3}

Species relative abundance at different classification, distance matrix heat map, and UPGMA clustering tree. a-f.The relative abundance of the top 10 species of genus in different taxonomic levels (phylum, class, order, family, genus, species). g.Index heat map of Beta diversity between groups. f. UPGMA clustering tree based on weighted unifrac distance at phylum. 